\title{
AVALIAÇÃO CINÉTICA DO BAGAÇO DE MALTE NA REMOÇÃO DO CORANTE AMARELO REATIVO
}

\author{
A. ZANUTTO ${ }^{1}$, B. C. da SILVA ${ }^{1}$, E. R. DUARTE ${ }^{1}$, L. N. B. de ALMEIDA ${ }^{1}$, J. M. T. A. \\ PIETROBELLI ${ }^{1}$ \\ ${ }^{1}$ Universidade Tecnológica Federal do Paraná, \\ E-mail para contato: jpietrobelli@utfpr.edu.br
}

\begin{abstract}
RESUMO - Este trabalho avaliou a cinética de remoção do corante amarelo reativo utilizando como biossorvente, o bagaço de malte, resíduo oriundo de cervejaria. Inicialmente foram realizados testes preliminares para avaliar: precipitação do corante, a influência do $\mathrm{pH}$ e do tamanho da partícula no processo de remoção. Os testes foram realizados em sistema de batelada com temperatura, agitação e $\mathrm{pH}$ controlados. Os resultados obtidos demonstraram que o tempo de equilíbrio foi atingido em torno de 6 horas com taxa de remoção do corante superior a 93\%. O modelo cinético de pseudo-segunda ordem foi o que melhor representou os dados experimentais. Os resultados obtidos demonstram que o bagaço de malte tem potencial para ser utilizada em sistemas de tratamento de efluentes têxteis.
\end{abstract}

\section{INTRODUÇÃ̃O}

A falta de controle efetivo na prevenção e tratamento dos resíduos tóxicos gerados por inúmeras indústrias pode representar uma ameaça aos recursos hídricos, vindo a comprometer a qualidade e a utilização destas águas.

A indústria têxtil representa um importante setor da economia, mas uma das principais características dos seus efluentes é a presença de cor, proveniente principalmente da etapa de tingimento. Esta etapa é responsável pela fixação do corante à fibra através de reações químicas e ao final da operação ocorre a lavagem para retirada do excesso de corante original ou hidrolisado não fixado à fibra nas etapas anteriores (GUARATINI E ZANONI, 2000).

Dentre as classes de corantes, os reativos são, mundialmente, bastante utilizados, apresentam como característica alta solubilidade em água e o estabelecimento de uma ligação covalente entre o corante e a fibra, conferindo maior estabilidade na cor do tecido tingido (GUARATINI E ZANONI, 2000). De acordo com Honorio (2013), os corantes reativos possuem grau de fixação de 60 a $90 \%$. Assim, o corante que não é fixado à fibra dos tecidos é descartado como efluente têxtil e pode contaminar corpos d'água e organismos vivos. A cor interfere na transmissão da luz solar dentro da água, prejudicando assim a fotossíntese das plantas. Além disso, a oxidação biológica desse material consome o oxigênio dissolvido existente no ambiente, prejudicando a atividade respiratória dos demais organismos ali presentes (SILVEIRA E SANTANNA, 1990). 
A biossorção é uma técnica alternativa no tratamento de efluentes têxteis para a remoção de corantes. Este método consiste na ligação de um componente presente em fase fluida (adsorvato) à um biossorvente (sólido) de origem natural. Para Honorio (2013), um biossorvente de baixo custo deve ser abundante na natureza, necessitar de poucos procedimentos ou ser um subproduto industrial.

De acordo com o site SEBRAE (2014), 13 bilhões de litros de cerveja foram produzidos no Brasil no ano de 2014, sendo o malte o principal ingrediente da cerveja. Segundo Brochier e Carvalho (2009), a cada $100 \mathrm{~kg}$ de malte obtém-se de 110 a $120 \mathrm{~kg}$ de resíduo úmido de cervejaria. O resíduo úmido de cervejaria é um subproduto do processo de fabricação da cerveja, também conhecido como bagaço de malte.

Tendo em vista que a literatura não apresenta estudos acerca do aproveitamento do bagaço de malte na remoção de poluentes, este trabalho visa preencher esta lacuna através da avaliação cinética de remoção do corante amarelo reativo pelo bagaço de malte.

\section{MATERIAIS E MÉTODOS}

\subsection{Preparo da biomassa, das soluções e da curva de calibração}

A biomassa utilizada nos ensaios foi cedida pela empresa Schultz Bier Microcervejaria, localizada na região de Ponta Grossa/PR. O resíduo foi seco a $30^{\circ} \mathrm{C}$ em estufa com circulação e renovação de ar (SL 102 - SOLAB). Em seguida, o resíduo foi triturado em macro moinho de facas, MA340 - Tipo Willey.

A solução sintética foi preparada a partir da dissolução do corante Amarelo Reafix B2R em água destilada. Para a obtenção do comprimento de onda no qual o corante Amarelo Reativo absorve a maior quantidade de luz, realizou-se uma varredura no espectrofotômetro UV-Vis Femto 800 XI com comprimento de onda na faixa de 300 a $1000 \mathrm{~nm}$ e solução de corante com concentração de $75 \mathrm{mg} \mathrm{L}^{-1}$. Em seguida, determinou-se uma curva de calibração utilizando soluções com concentração conhecida de 0 a $100 \mathrm{mg} \mathrm{L}^{-1}$. As concentrações de corante foram obtidas a partir da leitura das soluções em comprimento de onda obtido pela varredura, com auxílio da curva de calibração e utilização do espectrofotômetro UV-Vis Femto 800 XI. A quantidade de corante adsorvida pelo bagaço de malte foi calculada conforme o balanço de massa, expresso pela Equação 1.

$$
q=\frac{V\left(C_{0}-C_{t}\right)}{m_{s}}
$$

Em que, $q\left(\mathrm{mg} \mathrm{g}^{-1}\right)$ é a quantidade de corante adsorvida pelo bagaço, $C_{0}$ e $C$ as concentrações iniciais e finais do corante em solução $\left(\mathrm{mg} \mathrm{L}^{-1}\right), V$ o volume de solução (L) e $m$ a massa de adsorvente $(\mathrm{g})$.

\subsection{Testes preliminares}

O teste de precipitação teve por finalidade determinar o $\mathrm{pH}$ em que o corante apresentou precipitação significativa, fenômeno este que pode interferir na avaliação do processo de biossorção. O experimento foi realizado em duplicata, com solução de corante Amarelo 
Reativo a $75 \mathrm{mg} \mathrm{L}^{-1}$. Os ensaios de precipitação foram realizados em béqueres, com $50 \mathrm{~mL}$ da solução com pH ajustado individualmente $(1 ; 1,5 ; 2 ; 3 ; 4 ; 5 ; 6 ; 7 ; 8 ; 9 ; 10 ; 11 ; 12 ; 13 ; 14)$ através de soluções de hidróxido de sódio $(\mathrm{NaOH})$ e ácido clorídrico $(\mathrm{HCl})$. As soluções foram mantidas em repouso por 24 horas, à temperatura ambiente $\left( \pm 25^{\circ} \mathrm{C}\right)$, centrifugadas (280R - Excelsa) a $3000 \mathrm{rpm}$ por 10 minutos, e analisadas em espectrofotômetro UV-Vis.

Com a finalidade de verificar a influência da granulometria do bagaço de malte na remoção do corante, a biomassa seca foi peneirada, com o auxílio de um agitador eletromagnético (Bertel - AAKER) por 10 minutos. As faixas granulométricas avaliadas foram: $(16,20),(42,48),(500,635)$ mesh, correspondentes aos diâmetros médios de partícula de 0,$912 ; 0,323 ; 0,0225 \mathrm{~mm}$, além da mistura granulométrica. Para a realização do ensaio adicionou-se $50 \mathrm{~mL}$ de solução de corante amarelo reativo e $0,3 \mathrm{~g}$ de biomassa nos quatro tamanhos selecionados, separadamente, em erlenmeyers de $125 \mathrm{~mL}$. Em seguida, as amostras foram colocadas em incubadora com agitação rotativa, shaker (TE-420 - Tecnal), a uma velocidade de $100 \mathrm{rpm}$ e temperatura controlada de $30^{\circ} \mathrm{C}$, por 22 horas em pH 2 . Posteriormente, estas amostras foram centrifugadas e analisadas em relação à concentração de corante por meio de medidas em espectrofotômetro UV-vis.

O teste de $\mathrm{pH}$ tem por objetivo encontrar o $\mathrm{pH}$ ótimo para a remoção do corante pelo resíduo de cervejaria. Em erlenmeyer, adicionou-se 0,3 g de biomassa e $50 \mathrm{~mL}$ de solução do corante ajustada em pHs na faixa de 1 a 12. Em seguida, as amostras foram mantidas na incubadora shaker a $100 \mathrm{rpm}$ em $30^{\circ} \mathrm{C}$, por 22 horas. Após esse tempo, as amostras foram centrifugadas a $3000 \mathrm{rpm}$ por 10 minutos e a leitura realizada em espectrofotometro UV-Vis.

\subsection{Teste cinético}

O teste cinético consistiu na adição de $0,3 \mathrm{~g}$ de resíduo de cervejaria e $50 \mathrm{~mL}$ de solução de amarelo reativo $75 \mathrm{mg} \mathrm{L}^{-1} \mathrm{em}$ erlenmeyers de $125 \mathrm{~mL}$. As amostras foram colocadas sob agitação rotativa a $130 \mathrm{rpm}$ e $30^{\circ} \mathrm{C}$, e retiradas em intervalos de tempo pré-determinados (de 0 até 2880 minutos). Em seguida centrifugou-se as amostras (3000 rpm por $10 \mathrm{~min}$ ) e realizouse a leitura em UV-Vis.

\section{RESULTADOS E DISCUSSÃO}

\subsection{Testes preliminares}

Pelo teste de precipitação verificou-se que não houve precipitação significativa do corante nas faixas de $\mathrm{pH}$ analisadas, assim os valores das concentrações finais do corante não são influenciados por este fenômeno.

Em relação, a avaliação do efeito do $\mathrm{pH}$ na remoção do corante pela biomassa observouse um potencial de remoção próximo a $85 \%$ em pH 2, conforme segue na Figura 1. Por este motivo, o teste cinético foi conduzido neste valor de $\mathrm{pH}$, por ser o que mais favoreceu o desempenho da biossorção. Segundo Módenes et. al. (2011), com o decréscimo do pH o número de sítios ativos carregados negativamente na superfície do adsorvente diminui com o aumento prótons disponíveis na solução do corante. Desta forma, a adsorção de ânions é favorecida, uma vez que os corantes reativos possuem caráter aniônico devido à presença do grupo sulfonato $\left(-\mathrm{SO}_{3}{ }^{-}\right)$. 


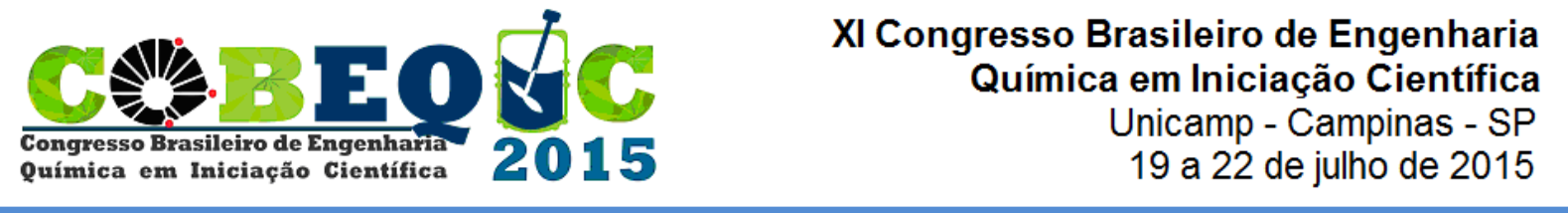

Figura 1: Influência do pH na remoção $\left(30^{\circ} \mathrm{C}\right.$ e $100 \mathrm{rpm} 22$ horas)

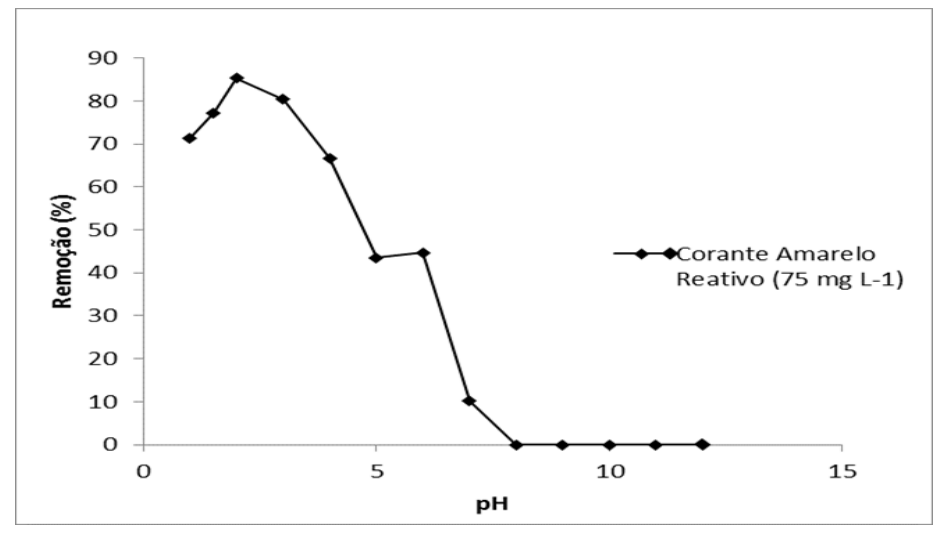

Quanto ao diâmetro de partícula, verificou-se um percentual de remoção de 78,41; 77,05; 77,78 e $85 \%$ para 0,$922 ; 0,323 ; 0,0225 \mathrm{~mm}$ e mistura granulométrica das partículas, respectivamente. Com isso observou-se que o diâmetro médio das partículas do bagaço não interferiu significativamente, no entanto observou-se uma maior porcentagem de remoção do corante pela mistura granulométrica (sem peneiramento). Assim, nos testes subsequentes foi utilizada mistura granulométrica de bagaço de malte seco, eliminando assim a etapa de peneiramento e realizando então o aproveitamento de todo bagaço disponível.

\subsection{Teste cinético}

Como apresentado na Figura 2, verificou-se uma redução superior a $60 \%$ na concentração do corante nos primeiros 10 minutos, atingindo o equilíbrio em torno de 360 minutos com remoção superior a 93\%.

Figura 2: Comportamento cinético do processo de biossorção (ensaio a $30^{\circ} \mathrm{C}, 130 \mathrm{rpm} \mathrm{e} \mathrm{pH} \mathrm{2)}$

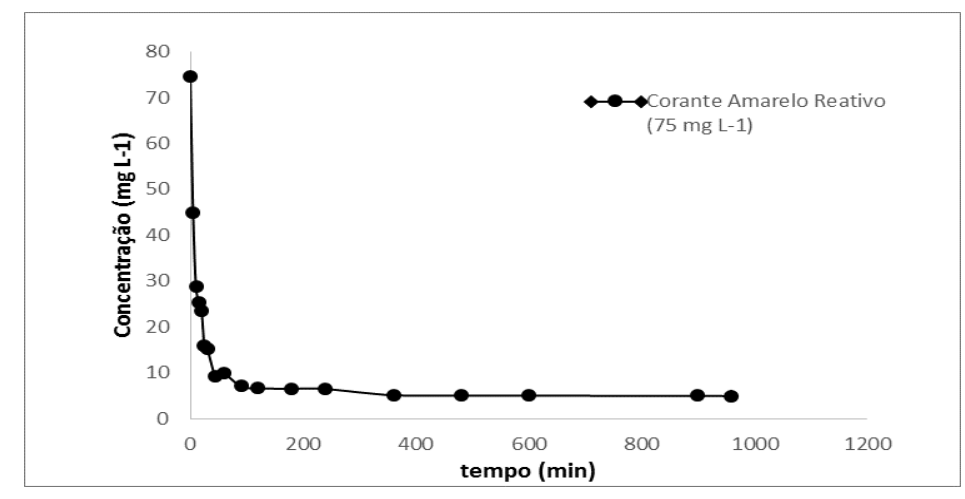

\subsection{Cinética de adsorção}

Para o estudo do comportamento cinético do sistema, comumente são utilizados modelos (apresentados na Tabela 1), entre os mais utilizados pode-se citar: modelo pseudoprimeira ordem, pseudo-segunda ordem e modelo de Elovich. O modelo de pseudo-primeira ordem refere-se à capacidade de adsorção do sólido. $\mathrm{O}$ modelo pseudo-segunda ordem também baseia-se na capacidade de adsorção do material adsorvente, no entanto, assume-se 
que o processo de adsorção é controlado pela adsorção química (ALVES, 2013). E o modelo de Elovich envolve o processo de quimiossorção, válido principalmente para sistemas onde a superfície do adsorvente é heterogênea (ROYER, 2008).

Realizaram-se os ajustes dos modelos cinéticos de adsorção supracitados aos dados experimentais de adsorção. Com isso, foi possível determinar os parâmetros e os coeficientes de correlação $\left(\mathrm{R}^{2}\right)$ para cada modelo, conforme segue na Tabela 1.

Tabela 1: Coeficientes de correlação e parâmetros de adsorção para os modelos cinéticos

\begin{tabular}{|c|c|c|}
\hline Modelo Cinético & Coeficiente de Correlação $\left(\mathrm{R}^{2}\right)$ & Parâmetros do modelo \\
\hline $\begin{array}{c}\text { Pseudo-primeira ordem: } \\
\log \left(q_{e q}-q_{t}\right)=\log \left(q_{e q}\right)-\left(\frac{K_{1}}{2,303}\right) t\end{array}$ & 0,8545 & $\begin{array}{l}\mathrm{K}_{1}=0,01543 \mathrm{~min}^{-1} \\
\mathrm{q}_{\mathrm{eq}}=3,4818 \mathrm{mg} \mathrm{L}^{-1}\end{array}$ \\
\hline $\begin{array}{l}\text { Pseudo-segunda ordem: } \\
\qquad \frac{1}{q_{t}}=\frac{1}{K_{2} q_{e q}^{2}}+\frac{1}{q_{e q}} t\end{array}$ & 0,9997 & $\begin{array}{c}\mathrm{K}_{2}=0,01758 \mathrm{~min}^{-1} \\
\mathrm{q}_{\mathrm{eq}}=11,7096 \mathrm{mg} \mathrm{L}^{-1}\end{array}$ \\
\hline $\begin{array}{c}\text { Elovich: } \\
q_{t}=\frac{1}{\beta} \ln (\alpha \beta)+\frac{1}{\beta} \ln (t)\end{array}$ & 0,7508 & $\begin{array}{c}\alpha=318,7189 \mathrm{mg} \mathrm{g}^{-1} \mathrm{~min}^{-1} \\
\beta=1,0026 \mathrm{~g} \mathrm{mg}^{-1}\end{array}$ \\
\hline
\end{tabular}

Através da Tabela 1 verifica-se que o modelo cinético que melhor representou os dados experimentais foi o modelo de Pseudo-segunda ordem. Assim como é verificado também na Figura 3, que apresenta a relação entre os modelos ajustados aos dados experimentais.

Figura 3: Modelos cinéticos para remoção do corante amarelo reativo $75 \mathrm{mg} \mathrm{L}^{-1}$

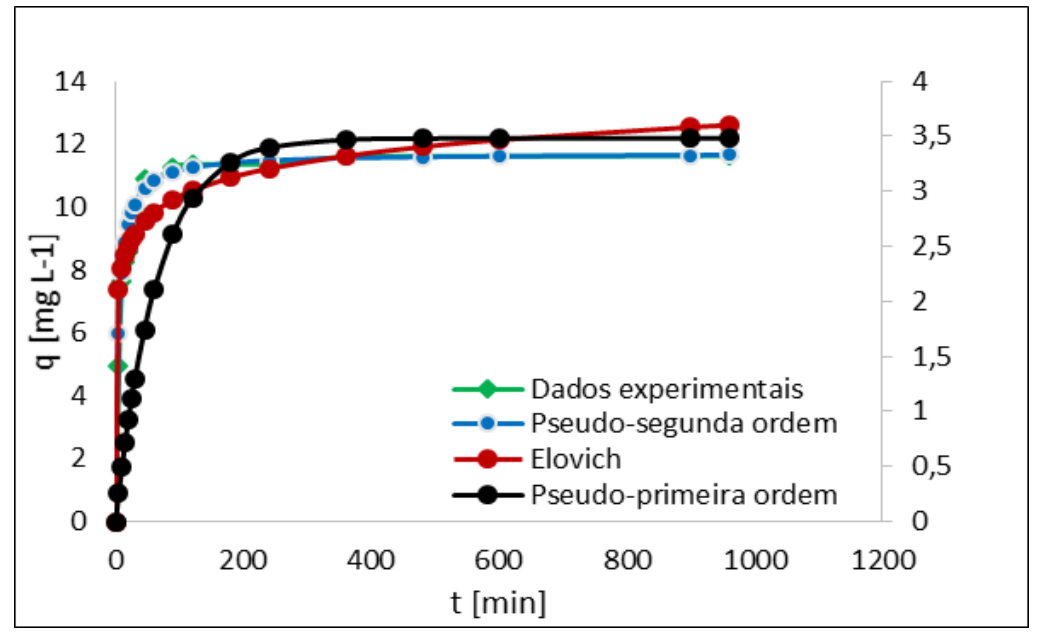

\section{CONCLUSÃO}

Nas condições testadas, verificou-se que o diâmetro médio das partículas do bagaço de malte não influenciou significativamente na remoção do corante. No entanto, confirmou-se a forte influência do $\mathrm{pH}$ neste processo, sendo que o $\mathrm{pH} 2$ foi considerado o ótimo na remoção do corante amarelo reativo, com valores superiores a $93 \%$ a $30^{\circ} \mathrm{C}$ e com rotação de $130 \mathrm{rpm}$. Os resultados dos experimentos revelam que o tempo de equilíbrio foi alcançado em 360 minutos, sendo o modelo de Pseudo-Segunda ordem o que melhor ajustou os dados cinéticos 


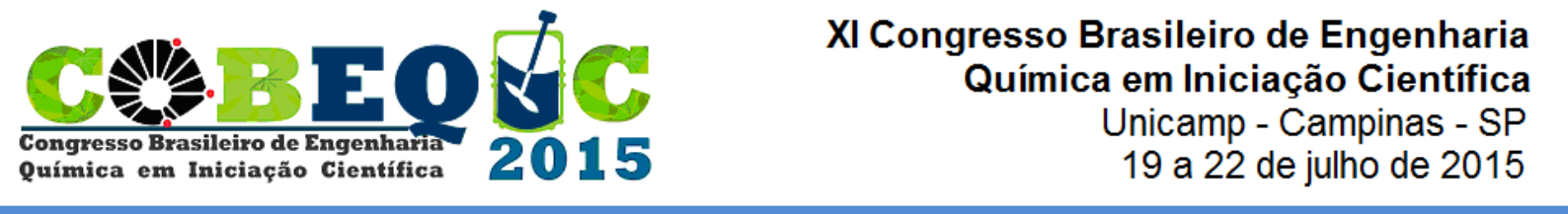

da biossorção pelo resíduo de cervejaria. Este modelo assume que o processo de adsorção é controlado pela adsorção química.

O bagaço de malte apresentou um elevado potencial no tratamento de efluentes têxteis contendo corante Amarelo Reativo, com altas taxas de remoção aliadas à elevada abundância e disponibilidade deste resíduo, a possibilidade de utilização sem nenhum tratamento prévio; a não ser a secagem, além da utilização de temperatura próxima da ambiente nos experimentos, o que facilita a reprodução dos mesmos em maior escala sem custo adicional de energia.

\section{NOMENCLATURA}

$\alpha \quad$ Taxa de adsorção inicial da equação cinética de Elovich ( $\left.\mathrm{mg} \mathrm{g}^{-1} \mathrm{~min}^{-1}\right)$

$\beta \quad$ Relação entre do grau de cobertura da superfície e a energia de ativação da equação cinética de Elovich $\left(\mathrm{g} \mathrm{mg}^{-1}\right)$

$K_{1} \quad$ Constante cinética do modelo de pseudo-primeira ordem $\left(\mathrm{min}^{-1}\right)$

$K_{2} \quad$ Constante cinética do modelo de pseudo-segunda ordem $\left(\mathrm{g} \mathrm{mg}^{-1} \mathrm{~min}^{-1}\right)$

\section{REFERÊNCIAS}

ALVES, F. C. Estudo dos processos de adsorção utilizando argilas como adsorventes para remoção do corante verde malaquita. 2013. 107 p. Dissertação (Mestrado) - Pós-graduação em Agroquímica, Universidade Federal de Lavras.

BROCHIER, M. A., CARVALHO, S. Aspectos ambientais, produtivos e econômicos do aproveitamento de resíduo úmido de cervejaria na alimentação de cordeiros em sistema de confinamento. Ciênc. agrotec. [online]. 2009, vol.33, n.5, pp. 1392-1399.

GUARATINI, C. C. I.; ZANONI, M.V.B. Corantes têxteis. Química Nova, São Paulo, v. 23, n. 1, p. 71-78, jan., 2000.

HONORIO, J. F. Emprego da casa de soja como adsorvente na remoção de corantes reativos têxteis. 2013. 114 f. Dissertação (Mestrado) - Pós-graduação em Engenharia Química, Universidade Estadual do Oeste do Paraná, Toledo.

MÓDENES, A. N. et al. UTILIZAÇÃO DA MACRÓFITA EGERIA DENSA NA BIOSORÇÃO DO CORANTE REATIVO 5G. Engevista, v. 3, n. 13, p.160-166, dez. 2011.

ROYER, B. Remoção de corantes têxteis utilizando casa de semente de Araucaria angustifolia como biossorvente. 2008. 68 p. Dissertação (Mestrado) - Pós-graduação em Química, Universidade Federal do Rio Grande do Sul.

SEBRAE. Consumo de cerveja no Brasil e no mundo. Resposta técnica. 2014.

SILVEIRA, S. S. B.; SANTANNA, F. S. P. Poluição Hídrica. In: MARGULIS, S. (Ed) Meio Ambiente: aspectos técnicos e econômicos. Brasília: PNDU/IPEA, p. 57-84, 1990. 